\title{
Some features of two-photon absorption in static electric field
}

\author{
V.M. Griban, O.V. Melnichuk \\ Mykola Gogol State Pedagogic University, 2 Kropyv'yans'kogo St., Nizhyn, 16600, Ukraine
}

\author{
L.M. Ovander \\ Engineering-Technological Institute, Zhytomyr, Ukraine
}

\section{E.F. Venger}

Institute of Semiconductor Physics, NAS Ukraine, 45 Prospect Nauki, Kyiv, 03028, Ukraine Phone: (38044) 26525 93; Fax: (38044) 26583 42; E-mail: mickle@semicond.kiev.ua

\begin{abstract}
We considered the effect of static uniform electric field on the process of two-photon excitonic absorption of light in crystals. The matrix element that determines the above process was calculated using a linear (in the photon field) operator of the electron-photon interaction within the framework of polariton theory. It involves a term that depends on the electric field strength. Using the group theory considerations, we obtained that , at definite orientations of the electric field and polarization vectors, two-photon absorption may occur due to the electric field effect only. The process probability depends also on both the crystal symmetry class and type of an excitonic state. Some special cases are considered in more detail. These are provided by the tables of angular dependencies of process probability, which enables one to experimentally check the results obtained.
\end{abstract}

Keywords: two-photon absorption, excitonic states, polariton, group theory considerations. Paper received 08.09.99; revised manuscript received 08.12.99; accepted for publication 14.03.00.

\section{Introduction}

Two-photon absorption is a nonlinear optical effect. It is as follows: excitations having energy $\hbar\left(\omega_{1}+\omega_{2}\right)$ appear in a quantum-mechanical system exposed to radiations whose frequencies are $\omega_{1}$ and $\omega_{2}$. (The specific case is when $\omega_{1}=\omega_{2}$.) The absorption intensity essentially depends on the system features. In what follows we shall deal with two-photon absorption in molecular crystals. The excitations appearing in them are considered to be Coulomb excitons.

It was shown in [1] that it is convenient to perform a correct theoretical analysis of nonlinear optical effects in crystals using a polariton model in which polaritons are a "mixture" of photons and Coulomb excitons. Three terms in the Hamiltonian of the "crystal + radiation field" system contribute to nonlinear effects in this model. They are the linear and square in the photon field terms in the operator of electron-photon interaction, $H_{i}^{(1)}$ and $H_{i}^{(2)}$, and operator of intermolecular Coulomb interaction, $H_{\text {Coul }}$.
The general theory of two-photon absorption was considered by a number of authors. A review of their papers was made in [2], so we shall not deal with it here. However, we would like to note the papers [3,4]. In [3] the anisotropy of two-photon absorption was considered with account for the term $H_{i}^{(1)}$, while in [4] the term $H_{i}^{(2)}$ was taken into account. The authors of [2] considered the twophoton absorption due to the term $H_{C o u l}$, i.e., to anharmonism in the Coulomb subsystem of crystal. They determined the dependence of two-photon absorption on the wave vector directions. The contributions from the above terms to the nonlinear optical effects were estimated in [1]. According to the estimations, one should expect that under conventional conditions the main contribution to the two-photon absorption can be provided by the term $H_{i}^{(1)}$.

An external static electric field exerts a substantial effect on the nonlinear optical phenomena. It was shown in [5] by one of the authors of this work that for crystals with center of inversion generation of the second optical 


\section{V.M. Griban et al.: Some features of two-photon absorption in static electric field.}

harmonic becomes possible in such fields. (In the absence of field, this generation is forbidden by the selection rules.) The authors of [6] investigated the sum harmonic generation in a static electric field under resonance, as well as some peculiarities of the tensor of nonlinear effects in this case.

Here we shall consider the effect of a uniform static electric field (hereafter referred to as electric field) on the two-photon absorption in crystals due to anharmonic components of the term $H_{i}^{(1)}$. For crystals with center of inversion placed in this field the two-photon transitions to antisymmetric final states become possible (in the absence of this field such transitions are forbidden). We shall determine also the angular dependencies (i.e., dependencies on the directions of polarization vectors and electric field vector) of the probability of this process in crystals of different symmetry classes for different final states.

In the approach used in this work two-photon absorption is treated as merging of two polaritons, with the frequency of the resulting polariton corresponding to the exciton absorption band. Our investigation is made using group theory, so the results obtained can be applied not only to molecular crystals.

\section{Matrix element of transition}

Let us assume that two polariton flows are produced in crystal exposed to external radiation. Their frequencies and polarization vectors are $\omega_{1}\left(\vec{k}_{1}\right), \omega_{2}\left(\vec{k}_{2}\right)$ and $\vec{e}_{1}, \vec{e}_{2}$, respectively. Using the technique discussed in [1], we shall determine the matrix element that corresponds to appearance of a Coulomb exciton whose energy is $E_{\mu}\left(\vec{k}_{1}+\vec{k}_{2}\right)=\hbar\left[\omega_{1}\left(\vec{k}_{1}\right)+\omega_{2}\left(\vec{k}_{2}\right)\right]$. To do this, one has to set off the operator $H_{i}^{(1)}$ the terms of the type $B_{\mu}^{+}\left(\vec{k}_{1}+\vec{k}_{2}\right) \cdot \xi_{\rho_{1}}\left(\vec{k}_{1}\right) \cdot \xi_{\rho 2}\left(\vec{k}_{2}\right)$. Here $B_{\mu}^{+}\left(\vec{k}_{1}+\vec{k}_{2}\right)$ is the creation operator for the Coulomb exciton with wave vector $\left(\vec{k}_{1}+\vec{k}_{2}\right)$ from the $\mu$-th band; $\xi_{\rho_{1}}\left(\vec{k}_{1}\right)$ and $\xi_{\rho_{2}}\left(\vec{k}_{2}\right)$ are the annihilation operators for polaritons with wave vectors $\vec{k}_{1}$ and $\vec{k}_{2}$ from the $\rho_{1}$-th and $\rho_{2}$-th branches, respectively. A coefficient at the product $B^{+} \xi \xi$ is the desired matrix element that corresponds to merging of polaritons with energies $\hbar \omega_{1}\left(\vec{k}_{1}\right), B^{+} \xi \xi$ and creation of an exciton whose energy is $E_{\mu}\left(\vec{k}_{1}+\vec{k}_{2}\right)$. Omitting cumbersome details of calculation, we write down the above matrix element (see also [1]):

$$
Q(\mu)=\frac{2 \pi \hbar}{V} \sqrt{\frac{N}{\omega_{1} \cdot \omega_{2}}} \cdot D_{1} \cdot D_{2} \cdot \alpha(\mu) .
$$

Here

$$
\alpha(\mu)=\sum_{j_{1}, j_{2}} \alpha_{j_{1} j_{2}}(\mu) \cdot e_{j_{1}} \cdot e_{j_{2}},
$$

$$
\begin{aligned}
\alpha_{j_{1} j_{2}}(\mu)= & \sum_{\mu_{1}}\left\{\frac{\left(0\left|j_{1}\right| \mu_{1}, \vec{k}_{1}\right)\left(\mu_{1}, \vec{k}_{1}\left|j_{2}\right| \mu, \vec{k}_{1}+\vec{k}_{2}\right)}{E_{\mu_{1}}\left(\vec{k}_{1}\right)-\hbar \omega_{1}}+\right. \\
& \left.+\frac{\left(0\left|j_{2}\right| \mu_{1}, \vec{k}_{2}\right)\left(\mu_{1}, \vec{k}_{2}\left|j_{1}\right| \mu, \vec{k}_{1}+\vec{k}_{2}\right)}{E_{\mu_{1}}\left(\vec{k}_{2}\right)-\hbar \omega_{2}}\right\} .
\end{aligned}
$$

The following notations are used in expressions (1)(3): $V$ is the crystal volume; $N$ is the number of unit cells; $\mu, \vec{k})$ is the wave function of a crystal with an excited Coulomb exciton; $\mid 0$ ) is the wave function of the crystal ground state; $e_{j_{1}}$ and $e_{j_{2}}$ are the projections of the polarization vectors, $\vec{e}_{1}$ and $\vec{e}_{2}$, on the coordinate axes (the labels $j_{1}, j_{2}$, are $\left.x, y, z\right) ; D_{1}$ and $D_{2}$ are of the following form:

$$
D_{i}=\frac{2 \cdot \sqrt{n_{i}}}{\left(n_{i}+1\right) \cdot A_{\rho_{i}}\left(\vec{k}_{i}\right)}
$$

Here $n_{i}(i=1,2)$ are the refractive indices; $A_{\rho_{i}}\left(\vec{k}_{i}\right)$ are the normalizing factors (they appear when the Hamiltonian is diagonalized [1]); $\left(\mu_{1}, \vec{k}_{1}\left|j_{n}\right| \mu_{2}, \vec{k}_{2}\right)$ is the matrix element of the operator $\frac{e}{m} I_{j_{n}}(e, m$ and $\vec{I}$ are the electron charge, mass and momentum operator, respectively).

$e_{j_{1}}$ and $e_{j_{2}}$ are the direction cosines of polarization vectors. In what follows we shall label them, according to [2-4], by $l_{1}, m_{1}, n_{1}$ (if $\left.j_{1}=x, y, z\right)$ or $l_{2}, m_{2}, n_{2}$ (if $\left.j_{2}=x, y, z\right)$.

Now let us determine the transition matrix element for a crystal placed in electric field whose strength is $\varepsilon$. In actual situations $\varepsilon<\varepsilon_{m o l}$, where $\varepsilon_{m o l}$ is the strength of the inner molecular electric field. Therefore, one can use the perturbation theory. It was shown in [5] that the wave function of the excitonic state of a crystal is as follows:

$$
\left.\mid \mu, \vec{k}, \vec{\varepsilon})=\mid \mu, \vec{k})-N \sum_{i, \mu^{\prime} \vec{k}^{\prime}} \varepsilon_{i} \frac{\left(\mu^{\prime}, \vec{k}^{\prime}\left|p_{i}\right| \mu, \vec{k}\right)}{E_{\mu}(\vec{k})-E_{\mu^{\prime}}\left(\vec{k}^{\prime}\right)} \mid \mu^{\prime}, \vec{k}^{\prime}\right) .
$$

Here $p_{i}$ are the components of the electric dipole moment of unit cell.

Replacing in expression (3) the wave functions $\mid \mu, \vec{k})$ by (5) and retaining the terms that are linear in the field $\vec{\varepsilon}$, one obtains, instead of $\alpha(\mu)$, the following expression:

$\alpha(\mu, \bar{\varepsilon})=\alpha(\mu)+\beta(\mu, \bar{\varepsilon})$

Here

$\beta(\mu, \varepsilon)=\sum_{j_{1}, j_{2}} \beta_{j_{1} j_{2}}(\mu, \varepsilon) \cdot e_{j_{1}} \cdot e_{j_{2}}$, 


$$
\begin{aligned}
& \beta_{j_{1} j_{2}}(\mu, \varepsilon)=-N \sum_{i} \varepsilon_{i} \cdot \gamma_{j_{1} j_{2} i}(\mu), \\
& \gamma_{j_{1} j_{2} i}(\mu)=\sum_{\mu_{1}, \mu^{\prime}, k^{\prime}}\left\{\frac{\left(0\left|j_{1}\right| \mu^{\prime}, \vec{k}^{\prime}\right)\left(\mu^{\prime}, \vec{k}^{\prime}\left|p_{i}\right| \mu_{1}, \vec{k}_{1}\right)\left(\mu_{1}, \vec{k}_{1}\left|j_{2}\right| \mu, \vec{k}_{1}+\vec{k}_{2}\right)}{\left[E_{\mu_{1}}\left(\vec{k}_{1}\right)-\hbar \omega_{1}\right] \cdot\left[E_{\mu_{1}}\left(\vec{k}_{1}\right)-E_{\mu^{\prime}}\left(\vec{k}^{\prime}\right)\right]}+\ldots\right\} .
\end{aligned}
$$

Five other similar terms in expression (9) are denoted by dots.

Therefore the matrix element that corresponds to an elementary act of two-photon absorption in a crystal placed in electric field may be written down as follows:

$$
Q(\mu, \bar{\varepsilon})=\frac{2 \pi \hbar}{V} \sqrt{\frac{N}{\omega_{1} \cdot \omega_{2}}} D_{1} D_{2}[\alpha(\mu)+\beta(\mu, \bar{\varepsilon})] .
$$

\section{Angular dependence of the probability of two-photon absorption in electric field}

An important characteristic of two-photon absorption is the dependence of its probability on the direction cosines of polarization vectors and (in our case) on the sense of electric field $\varepsilon$. The probability of two polaritons merging with creation of a Coulomb exciton is proportional to the squared magnitude of the matrix element (10). Therefore, taking into account expressions (2) and (7), one can write down:

$$
W(\mu, \bar{\varepsilon}) \sim\left|\sum_{j_{1}, j_{2}}\left[\alpha_{j_{1} j_{2}}(\mu)+\beta_{j_{1} j_{2}}(\mu, \bar{\varepsilon})\right] \cdot e_{j_{1}} \cdot e_{j_{2}}\right|^{2} .
$$

To separate the angular dependence out of (11), one has to determine the tensors $\alpha(\mu)$ and $\beta(\mu, \vec{\varepsilon})$, i.e., to find the non-zero components and interrelations between them. To this end we shall use the group theory approach.

In the optical spectral region wave vectors are small. Therefore, let us assume that, approximately, $\vec{k}_{1}, \vec{k}_{2} \rightarrow 0$. Then the excitonic states can be classified according to the irreducible representations of the point symmetry group of a crystal $[1,3]$. This means that the wave functions $\mid \mu, \vec{k})$ realize the corresponding irreducible representations of the point symmetry group.

Returning to expressions (3) and (9), one should note that the ground state $\mid 0$ ) and the quantities of the type

$$
\sum_{\mu} \frac{\mid \mu, \vec{k})(\mu, \vec{k} \mid}{E_{\mu}(k)-\hbar \omega} \text { are fully symmetric [1,2], so isomor- }
$$

phism exists for the tensors $\alpha(\mu)$ and $\gamma(\mu)$ :

$$
\begin{aligned}
& \alpha_{j_{1} j_{2}}(\mu) \rightarrow\left(j_{1} j_{2}\right)_{\mu}, \\
& \gamma_{j_{1} j_{2} i}(\mu) \rightarrow\left(j_{1} j_{2} i\right)_{\mu} .
\end{aligned}
$$

This means that the right- and left-hand sides of expressions (12) and (13) have the same transformation prop- erties under symmetry operations. The index $m$ in the righthand sides of the above expressions indicates at projection of the products of coordinates, $\left(j_{1} j_{2}\right)$ and $\left(j_{1} j_{2} i\right)$, onto the irreducible representation according to which the final state wave function, $\left.\mid \mu, \vec{k}_{1}+\vec{k}_{2}\right)$, transforms [7].

Having determined the tensors $\alpha(\mu)$ and $\gamma(\mu)$ (employing expressions (12)-(15)) and then tensor $\beta(\mu, \vec{\varepsilon})$ (employing expression (8)), let us determine the angular dependence of the function $W(\mu, \vec{\varepsilon})$ using expression (11). This dependence, however, is too complicated. Besides, in actual conditions $\beta(\mu, \bar{\varepsilon})<<\alpha(\mu)$, and so presence of the term $\alpha(\mu)$ makes it difficult to study the effects resulting from $\beta(\mu, \bar{\varepsilon})$. And, at last, it is of importance to know how the results obtained could be checked experimentally. In this connection, let us clarify the conditions under which two-photon absorption occurs due to the effect of electric field (i.e., the term $\beta(\mu, \vec{\varepsilon})$ in (11)) only. In this case

$W(\mu, \varepsilon) \sim\left|\sum_{j_{1}, j_{2}} \beta_{j_{1} j_{2}}(\mu, \varepsilon) \cdot e_{j_{1}} \cdot e_{j_{2}}\right|^{2}$.

These conditions mean that $\alpha(\mu)=0$, the operators $H_{i}^{(2)}$ and $H_{C o u l}$ do not contribute to the process and, finally, optical activity does not cause any complications.

The above conditions could be satisfied if one deals with crystals having center of inversion. In this case the double and triple products of coordinates transform according to different irreducible representations of the symmetry group. The double products transform according to the representations of $g$-type $\left(A_{g}, B_{g}, \ldots\right)$ that are symmetric under inversion, while the triple ones transform according to the representations of $u$-type $\left(A_{u}, B_{u}, \ldots\right)$ that are antisymmetric under inversion. According to expression (12), this means that at two-photon absorption with transition to the antisymmetric final states the term $\alpha(\mu)$ is zero. In this case the contribution from the operator $H_{\text {Coul }}$ is also zero [2]. Crystals that have center of inversion are optically inactive. And, at last, to eliminate the effect coming from the operator $H_{i}^{(2)}$, it is sufficient to choose polarization vectors that are normal to each other $[1,2]$.

With the above considerations, let us illustrate the results obtained by the case of two-photon absorption in a crystal of class $D_{4 h}(4 / \mathrm{mmm})$. The symmetry group $D_{4 h}$ has four symmetric one-dimensional $(1 D)$ irreducible representations ( $A_{1 g}, B_{1 g}, A_{2 g}, B_{2 g}$ ), four antisymmetric $1 D$ irreducible representations $\left(A_{1 u}, B_{1 u}, A_{2 u}, B_{2 u}\right)$ and two $2 D$ irreducible representations $\left(E_{g}, E_{u}\right)$. The antisymmet- 


\section{V.M. Griban et al.: Some features of two-photon absorption in static electric field.}

ric representation $E_{u}$ has two rows, $E_{u}(x)$ and $E_{u}(y)$, according to which the coordinates $x$ and $y$ transform [7].

The double products of coordinates transform according to the irreducible representations $A_{1 u}, A_{2 g}, B_{1 u}, B_{2 g}$, $E_{g}$. Therefore, the two-photon transitions to excitonic states of these types provide a contribution from the term $\alpha(\mu)$ to the process. And a contribution from the term $\beta(\mu, \vec{\varepsilon})$ is provided by the transitions to the final states of the $A_{1 u^{-}}, B_{1 u^{-}}, A_{2 u^{-}}, B_{2 u^{-}}, E_{u}(x)$ - and $E_{u}(y)$-types. The triple products of coordinates transform according to these irreducible representations - see Table 1. The corresponding calculations (that are omitted here) were performed with the technique presented in $[7,8]$.

Now let us suppose that the final excitonic state, $\left.\mid \mu, \vec{k}_{1}+\vec{k}_{2}\right)$, belongs to the $A_{1 u}$-type. Then, using expression (13) and Table 1, one can write down the following relations:

$$
\begin{aligned}
& \gamma_{y z x}\left(A_{1 u}\right)=-\gamma_{x z y}\left(A_{1 u}\right)=a_{1}, \\
& \gamma_{z y x}\left(A_{1 u}\right)=-\gamma_{z x y}\left(A_{1 u}\right)=a_{2}, \\
& \gamma_{x y z}\left(A_{1 u}\right)=-\gamma_{y x z}\left(A_{1 u}\right)=a_{3} .
\end{aligned}
$$

The other components of tensor $g(m)$ are zero in our case.

Inserting (15) to expression (8), the resulting components of tensor $\beta(\mu, \varepsilon)$ to expression (14) and factoring$N a_{1}$ out, one gets the angular dependence of the probability of two-photon absorption due to transitions to the excitonic states of the $A_{1}$-type in electric field:

$$
\begin{aligned}
& \Phi\left(A_{1 u}, \vec{\varepsilon}\right)=\left[\left(m_{1} n_{2}+\lambda_{1} n_{1} m_{2}\right) \varepsilon_{x}-\right. \\
& \left.\left(l_{1} n_{2}+\lambda_{1} n_{1} l_{2}\right) \varepsilon_{y}+\lambda_{2}\left(l_{1} m_{2}-m_{1} l_{2}\right) \varepsilon_{z}\right]^{2} .
\end{aligned}
$$

Here $\lambda_{1}=a_{2} / a_{1}, \lambda_{2}=a_{3} / a_{1}$.

In this way one can obtain the angular dependencies of the process probabilities for other types of final excitonic states in crystals whose symmetry is $D_{4 h}$ (as well as in crystals belonging to other symmetry classes). The results of calculations are presented in Table 2. They are rather cumbersome. That is why we have restricted ourselves to the crystal classes $D_{4 h}, C_{6 h}$ and $S_{6}$ that belong to the tetragonal, hexagonal and trigonal systems. For the sake of simplicity, we do not label the coefficients $1_{i}$ by $m$ (i.e., one should remember that $\lambda_{1}\left(A_{1 u}\right) \neq \lambda_{1}\left(A_{2 u}\right)$, etc. $)$.

\section{Discussion of results obtained}

Some considerations that follow may serve as an evidence that the results obtained are correct.

It was noted above that in actual conditions $\varepsilon<<\varepsilon_{\text {mol }}$, and so electric field may be treated as a weak perturbation. In this case the excitonic states are classified as in the absence of electric field, i.e., according to the irreducible representations of the point group of crystal symmetry.

Now let us discuss a hypothetical case of strong electric field when the above condition breaks down. In this case, electric field is to be considered as part of the system studied, and the operator of its interaction with the electrons of the crystal is to be included to the zero-approximation Hamiltonian.

Uniform electric field is characterized by the axial symmetry only. Therefore the symmetry of a new system "crystal + radiation field + electric field" is, as a rule, lower than the crystal symmetry. In this case the excitonic states are to be classified according to the irreducible representations of a new symmetry group. The angular dependence of two-photon absorption is now determined by the term $\alpha(\mu)$ in expression (11).

Let us suppose that such a strong electric field is directed along the $z$-axis. In this case only two symmetry elements exist in a crystal of the $D_{4 h}$ symmetry, namely, the unit element, $E$, and axis of revolution, $C_{4}$. The symmetry group of the new system is $C_{4}$. This symmetry group has two $1 D$ irreducible representations, $A$ and $B$, and one $2 D$ irreducible representation, $E$. Using expressions (11) and (12) and taking into account that now $\beta(\mu, \vec{\varepsilon})=0$, one can obtain the following angular dependencies for the probability of two-photon absorption (see also [3]):

$$
\begin{aligned}
& \Phi(A)=\left[l_{1} l_{2}+m_{1} m_{2}+\lambda_{1}\left(l_{1} m_{2}-m_{1} l_{2}\right)+\lambda_{2} n_{1} n_{2}\right]^{2}, \\
& \Phi(B)=\left[l_{1} l_{2}-m_{1} m_{2}+\lambda\left(l_{1} m_{2}+m_{1} l_{2}\right)\right]^{2}, \\
& \left.\Phi(E)=\left[l_{1} n_{2}+\lambda_{1} n_{1} l_{2}+\lambda_{2} m_{1} n_{2}+\lambda_{3} n_{1} m_{2}\right)\right]^{2} .
\end{aligned}
$$

\begin{tabular}{|c|c|c|c|c|c|c|c|}
\hline$\mu$ & \multicolumn{7}{|c|}{ Products of coordinates } \\
\hline$A_{1 u}$ & \multicolumn{2}{|c|}{$x_{1} y_{2} z_{3}-y_{1} x_{2} z_{3}$} & \multicolumn{2}{|c|}{$x_{1} z_{2} y_{3}-y_{1} z_{2} x_{3}$} & \multicolumn{2}{|c|}{$z_{1} x_{2} y_{3}-z_{1} y_{2} x_{3}$} & \\
\hline$A_{2 u}$ & \multicolumn{2}{|c|}{$x_{1} x_{2} z_{3}+y_{1} y_{2} z_{3}$} & \multicolumn{2}{|c|}{$x_{1} z_{2} x_{3}+y_{1} z_{2} y_{3}$} & \multicolumn{2}{|c|}{$z_{1} x_{2} x_{3}+z_{1} y_{2} y_{3}$} & $z_{1} z_{2} z_{3}$ \\
\hline$B_{1 u}$ & \multicolumn{2}{|c|}{$x_{1} y_{2} z_{3}+y_{1} x_{2} z_{3}$} & \multicolumn{2}{|c|}{$x_{1} z_{2} y_{3}+y_{1} z_{2} x_{3}$} & \multicolumn{2}{|c|}{$z_{1} x_{2} y_{3}+z_{1} y_{2} x_{3}$} & \\
\hline$B_{2 u}$ & \multicolumn{2}{|c|}{$x_{1} x_{2} z_{3}-y_{1} y_{2} z_{3}$} & \multicolumn{2}{|c|}{$x_{1} z_{2} x_{3}-y_{1} z_{2} y_{3}$} & \multicolumn{2}{|c|}{$z_{1} x_{2} x_{3}-z_{1} y_{2} y_{3}$} & \\
\hline$E_{u}(x)$ & $x_{1} x_{2} x_{3}$ & $y_{1} y_{2} x_{3}$ & $y_{1} x_{2} y_{3}$ & $x_{1} y_{2} y_{3}$ & $z_{1} z_{2} x_{3}$ & $z_{1} x_{2} z_{3}$ & $x_{1} z_{2} z_{3}$ \\
\hline$E_{u}(y)$ & $y_{1} y_{2} y_{3}$ & $x_{1} x_{2} y_{3}$ & $x_{1} y_{2} x_{3}$ & $y_{1} x_{2} x_{3}$ & $z_{1} z_{2} y_{3}$ & $z_{1} y_{2} z_{3}$ & $y_{1} z_{2} z_{3}$ \\
\hline
\end{tabular}

A comparison of these angular dependencies with those presented in Table 2 for the class $D_{4 h}$ in the case when $\mathrm{e}_{x}=\mathrm{e}_{y}=0$ indicates at interrelations between them:

Table 1.Triple products of coordinates that transform according to the irreducible representations of the $D_{4 h}$ group 
V.M. Griban et al.: Some features of two-photon absorption in static electric field.

Table 2. Angular dependencies of the two-photon absorption probabilities in crystals of different symmetry

\begin{tabular}{|c|c|c|}
\hline Class & $\mu$ & $\Phi(\mu, \vec{\varepsilon})$ \\
\hline \multirow{6}{*}{$D_{4 h}$} & $A_{1 u}$ & {$\left[\left(m_{1} n_{2}+\lambda_{1} n_{1} m_{2}\right) \varepsilon_{x}-\left(l_{1} n_{2}+\lambda_{1} n_{1} l_{2}\right) \varepsilon_{y}+\lambda_{2}\left(l_{1} m_{2}-m_{1} l_{2}\right) \varepsilon_{z}\right]^{2}$} \\
\hline & $\mathrm{A}_{2 u}$ & {$\left[\left(n_{1} l_{2}+\lambda_{1} l_{1} n_{2}\right) \varepsilon_{x}+\left(n_{1} m_{2}+\lambda_{1} m_{1} n_{2}\right) \varepsilon_{y}+\left(\lambda_{2} l_{1} l_{2}+\lambda_{2} m_{1} m_{2}+\lambda_{3} n_{1} n_{2}\right) \varepsilon_{z}\right]^{2}$} \\
\hline & $B_{1 u}$ & {$\left[\left(m_{1} n_{2}+\lambda_{1} n_{1} m_{2}\right) \varepsilon_{x}+\left(l_{1} n_{2}+\lambda_{1} n_{1} l_{2}\right) \varepsilon_{y}+\lambda_{2}\left(l_{1} m_{2}+m_{1} l_{2}\right) \varepsilon_{z}\right]^{2}$} \\
\hline & $B_{2 u}$ & {$\left[\left(l_{1} n_{2}+\lambda_{1} n_{1} l_{2}\right) \varepsilon_{x}-\left(m_{1} n_{2}+\lambda_{1} n_{1} m_{2}\right) \varepsilon_{y}+\lambda_{2}\left(l_{1} l_{2}-m_{1} m_{2}\right) \varepsilon_{z}\right]^{2}$} \\
\hline & $E_{u}(x)$ & {$\left[\left(l_{1} l_{2}+\lambda_{1} m_{1} m_{2}+\lambda_{2} n_{1} n_{2}\right) \varepsilon_{x}+\left(\lambda_{3} l_{1} m_{2}+\lambda_{4} m_{1} l_{2}\right) \varepsilon_{y}+\left(\lambda_{5} l_{1} n_{2}+\lambda_{6} n_{1} l_{2}\right) \varepsilon_{z}\right]^{2}$} \\
\hline & $E_{u}(y)$ & {$\left[\left(\lambda_{3} l_{1} m_{2}+\lambda_{4} m_{1} l_{2}\right) \varepsilon_{x}+\left(l_{1} l_{2}+\lambda_{1} m_{1} m_{2}+\lambda_{2} n_{1} n_{2}\right) \varepsilon_{y}+\left(\lambda_{5} m_{1} n_{2}+\lambda_{6} n_{1} m_{2}\right) \varepsilon_{z}\right]^{2}$} \\
\hline \multirow{6}{*}{$C_{6 h}$} & $A_{u}$ & $\left\lfloor\left(l_{1} n_{2}+\lambda_{1} n_{1} l_{2}+\lambda_{2} m_{1} n_{2}+\lambda_{3} n_{1} m_{2}\right) \varepsilon_{x}+\left(m_{1} n_{2}+\lambda_{1} n_{1} m_{2}-\lambda_{2} l_{1} n_{2}-\lambda_{3} n_{1} l_{2}\right) \varepsilon_{y}+\right.$ \\
\hline & & $\left.+\left(\lambda_{4} l_{1} l_{2}+\lambda_{4} m_{1} m_{2}+\lambda_{5} m_{1} l_{2}-\lambda_{5} l_{1} m_{2}+\lambda_{6} n_{1} n_{2}\right) \varepsilon_{z}\right]^{2}$ \\
\hline & $E_{1 u}$ & {$\left[\left(l_{1} n_{2}+\lambda_{1} n_{1} l_{2}+\lambda_{2} m_{1} n_{2}+\lambda_{3} n_{1} m_{2}\right) \varepsilon_{x}-\left(m_{1} n_{2}+\lambda_{1} n_{1} m_{2}-\lambda_{2} l_{1} n_{2}-\lambda_{3} n_{1} l_{2}\right) \varepsilon_{y}+\right.$} \\
\hline & & $\left.+\left(\lambda_{4} l_{1} l_{2}-\lambda_{4} m_{1} m_{2}+\lambda_{5} l_{1} m_{2}+\lambda_{5} m_{1} l_{2}\right) \varepsilon_{z}\right]^{2}$ \\
\hline & $E_{2 u}$ & $\left\lfloor\left(l_{1} l_{2}+m_{1} m_{1}+\lambda_{1} l_{1} m_{2}+\lambda_{1} m_{1} l_{2}+\lambda_{2} n_{1} n_{2}\right) \varepsilon_{x}+\left(l_{1} m_{2}+m_{1} l_{2}+\lambda_{1} l_{1} l_{2}+\lambda_{1} m_{1} m_{2}+\lambda_{3} n_{1} n_{2}\right) \varepsilon_{y}+\right.$ \\
\hline & & $\left.+\left(\lambda_{4} l_{1} n_{2}+\lambda_{5} n_{1} l_{2}+\lambda_{6} m_{1} n_{2}+\lambda_{7} n_{1} m_{2}\right) \varepsilon_{z}\right]^{2}$ \\
\hline \multirow[t]{4}{*}{$S_{6}$} & $A_{u}$ & $\left\lfloor\left(l_{1} n_{2}+\lambda_{1} n_{1} l_{2}+\lambda_{2} m_{1} n_{2}+\lambda_{3} n_{1} m_{2}\right) \varepsilon_{x}+\left(m_{1} n_{2}+\lambda_{1} n_{1} m_{2}-\lambda_{2} l_{1} n_{2}-\lambda_{3} n_{1} l_{2}\right) \varepsilon_{y}+\right.$ \\
\hline & & $\left.+\left(\lambda_{4} l_{1} l_{2}+\lambda_{4} m_{1} m_{2}+\lambda_{5} n_{1} n_{2}+\lambda_{6} l_{1} m_{2}-\lambda_{6} m_{1} l_{2}\right) \varepsilon_{z}\right]^{2}$ \\
\hline & $E_{u}$ & {$\left[\left(l_{1} l_{2}+m_{1} m_{2}+\lambda_{1} n_{1} n_{2}+\lambda_{2} l_{1} m_{2}+\lambda_{3} m_{1} l_{2}\right) \varepsilon_{x}+\left(l_{1} l_{2}+m_{1} m_{2}+\lambda_{4} n_{1} n_{2}+\right.\right.$} \\
\hline & & $\left.\left.+\lambda_{2} m_{1} l_{2}+\lambda_{3} l_{1} m_{2}\right) \varepsilon_{y}+\left(\lambda_{5} l_{1} n_{2}+\lambda_{6} n_{1} l_{2}+\lambda_{7} m_{1} n_{2}+\lambda_{8} n_{1} m_{2}\right) \varepsilon_{z}\right]^{2}$ \\
\hline
\end{tabular}

$\Phi(A)=\Phi\left(A_{1 u}, \varepsilon_{z}\right)+\Phi\left(A_{2 u}, \varepsilon_{z}\right)$,

$\Phi(B)=\Phi\left(B_{1 u}, \varepsilon_{z}\right)+\Phi\left(B_{2 u}, \varepsilon_{z}\right)$,

$\Phi(E)=\Phi\left(E_{u}(x), \varepsilon_{z}\right)+\Phi\left(E_{u}(y), \varepsilon_{z}\right) \cdot$

If electric field is switched off (i.e., the symmetry of the system studied changes from $C_{4}$ to $D_{4 h}$ ), then the excitonic state $A(B)$ of the group $C_{4}$ splits into the $A_{1 u}$ - and $A_{2 u}$ - type ( $B_{1 u^{-}}$and $B_{2 u}$-type) states of the group $D_{4 h}$, etc.

If electric field is directed along the $x$-axis, then the system symmetry group is $C_{s}$. Its irreducible representations are $A^{\prime}$ and $A^{\prime \prime}$ [7]. One can show that in this case

$$
\begin{aligned}
& \Phi\left(A^{\prime}\right)=\Phi\left(A_{1 u}, \varepsilon_{z}\right)+\Phi\left(A_{2 u}, \varepsilon_{x}\right)+ \\
& +\Phi\left(B_{1 u}, \varepsilon_{x}\right)+\Phi\left(B_{2 u}, \varepsilon_{x}\right) \\
& \Phi\left(A^{\prime \prime}\right)=\Phi\left(E_{u}(x), \varepsilon_{x}\right)+\Phi\left(E_{u}(y), \varepsilon_{x}\right) \\
& +\Phi\left(B_{1 u}, \varepsilon_{x}\right)+\Phi\left(B_{2 u}, \varepsilon_{x}\right)
\end{aligned}
$$

If electric field is directed along the $y$-axis, then the result is the same, except that $\mathrm{e}_{y}$ is substituted for $\mathrm{e}_{x}$ in expressions (23), (24).
In conclusion, let us discuss a possible experimental situation. Let us consider a crystal (belonging to symmetry class $D_{4 h}$ ) that is cut out as a rectangular parallelepiped along the optical $z$-axis, with its side faces normal to the $x$ - and $y$-axes. Its excitonic states $E_{u}(x)$ and $E_{u}(y)$ of frequency $\omega$ are considered to be known. This crystal is placed in electric field directed along the $z$-axis.

Let us assume that two linearly polarized light rays are incident on this crystal. One of them, of frequency $\omega_{1}$, is $y$-polarized and directed along the $x$-axis. Another ray, of frequency $\omega_{2}$, is $z$-polarized and directed along the $y$ axis. In this case two polariton flows will appear in the crystal. They correspond to an ordinary ray, of frequency $\omega_{1}$, for which $m_{1}=1, l_{1}=n_{1}=0$, and extraordinary ray, of frequency $\omega_{2}$, for which $n_{2}=1, l_{2}=m_{2}=0$. If $\omega_{1}+\omega_{2}$ $=\omega$, then, according to Table 2 , two-photon absorption will occur only due to the two-photon transitions to the $E_{u}(y)$-type excitonic state. If the ray that is directed along the $x$-axis is $z$-polarized and that directed along the $y$ axis is $x$-polarized, then the transitions to the $E_{u}(x)$-type excitonic state are possible. If electric field is oriented along the $x$ - or $y$-axis, then two-photon absorption will occur due to transitions to the $A_{1 u^{-}}$and $B_{1 u^{-}}$type (or $A_{2 u^{-}}$ and $B_{2 u^{-}}$type) excitonic states. 


\section{V.M. Griban et al.: Some features of two-photon absorption in static electric field.}

\section{Conclusion}

We have shown that the interaction between crystal and electric field results in appearance of a term (that is linear in the electric field strength) in the matrix element that determines two-photon excitonic absorption of light. This term makes possible the two-photon transitions that were forbidden by selection rules. The above term determines angular dependence of the process probability that differs from that in the absence of electric field.

For crystals with center of inversion some special cases are discussed when two-photon absorption occurs only due to the effect of electric field accompanied by crystal transition to antisymmetric excitonic states. The tables of the above angular dependencies are presented for some crystal symmetry classes. It is shown that there exists a possibility to experimentally check the results obtained and study the excitonic state types.

\section{References}

1. L.N. Ovander, Nonlinear optical effects in crystals (in Russian) // Uspekhi Fiz. Nauk 86 (1), pp. 1-39 (1965).

2. V.M. Griban, L.N. Ovander, Contribution from anharmonism to the two-photon absorption in a Coulomb subsystem (in Russian) // Fiz. Tverd. Tela 12 (2), pp. 448-454 (1970).

3. M. Inoue, Y. Toyozawa, Two-photon absorption and energy band structure // J. Phys. Soc. Japan 20 (3) pp. 363-374,(1965).

4. A.I. Bobrysheva, S.A. Moskalenko, On the two-photon induced luminescence in intrinsic semiconductors and insulators. Transition from an excitonic state (in Russian) // Izv. $A N$ SSSR, Ser. Phys. 30 (9), pp. 1539-1541 (1966).

5. V.M. Griban, On sum harmonic generation in crystals with center of inversion (in Russian) // Izv. Vuzov SSSR. Fizika $\mathbf{N}$ 6, pp.107-110 (1969).

6. E.F. Venger, V.M. Griban, A.V. Melnichuk, Resonance generation of sum harmonic in static electric field // Semiconductor Physics, Quantum Electronics \& Optoelectronics 2 (3), pp. 98-102 (1999).

7. L.D. Landau, E.M. Lifshits, Quantum Mechanics (in Russian), p.443, Nauka, Moscow (1989).

8. M.I. Petrashen, E.D. Trifonov, Group Theory Applications in Quantum Mechanics (in Russian), Nauka, Moscow (1967). 